\title{
Imaginal desensitisation plus motivational interviewing for pathological gambling: randomised controlled trial
}

Jon E. Grant, Christopher B. Donahue, Brian L. Odlaug, Suck Won Kim, Michael J. Miller and Nancy M. Petry

\author{
Summary \\ Sixty-eight individuals were randomised to either six \\ sessions of imaginal desensitisation plus motivational \\ interviewing (IDMI) or Gamblers Anonymous. Individuals \\ assigned to IDMI had significantly greater reductions in \\ Yale-Brown Obsessive Compulsive Scale Modified for \\ Pathological Gambling total scores, gambling urges and \\ gambling behaviour. People who failed to respond to \\ Gamblers Anonymous reported significantly greater reduction \\ in pathological gambling symptoms following later
}

assignment to IDMI. Abstinence was achieved by $63.6 \%$ during the acute IDMI treatment period.

\section{Declaration of interest}

J.E.G. has received research grants from Forest

Pharmaceuticals, GlaxoSmithKline and Somaxon Pharmaceuticals he has been a consultant to Somaxon Pharmaceuticals and for law offices as an expert in pathological gambling.
Pathological gambling is characterised by persistent and recurrent maladaptive patterns of gambling behaviour affecting from 0.4 to $1.6 \%$ of individuals worldwide. ${ }^{1}$ Studies suggest that cognitivebehavioural interventions, motivational interviewing and imaginal desensitisation may be beneficial for pathological gambling. ${ }^{1,2}$ This study sought to combine the strengths of prior treatments into a brief, 6-session format of imaginal desensitisation plus motivational interviewing (IDMI). We hypothesised it would reduce the severity of pathological gambling symptoms to a greater extent than referral to Gamblers Anonymous (GA).

\section{Method}

Men and women aged 18 to 75 with pathological gambling ${ }^{3}$ and who had gambled at least once per week for the past 2 months were included. Exclusion criteria included: past 3-month substance use disorder; positive urine drug screen at screening; current psychotherapy or medication for pathological gambling; previous GA attendance; suicidal intentions; and current use of psychotropic medications. The University of Minnesota's institutional review board approved the study and informed consent. After complete description of the study, participants provided written informed consent. This study is registered at www.clinicaltrials.gov: NCT00337753.

Manualised IDMI consisted of six sessions, each lasting $1 \mathrm{~h}$, over an 8-week period. Session one consisted of psychoeducation and motivational enhancement. Session two focused on functional analysis and behavioural strategies. Session three focused on coping with gambling urges and changing irrational thinking. Session four introduced imaginal desensitisation by creating and audiotaping three gambling scenarios that stimulated gambling urges. Relaxation training and cognitive skills were used to cope with the urges that the scenarios elicited. Participants were instructed to listen to the tape three times each day (reported mean 2.2 times (s.d. $=0.8)$ ). Session five included relapse prevention and assertiveness training. Session six included significantother involvement, education and therapy.

Participants in the GA group received a list of meeting times and locations for 75 GA meetings throughout the Twin Cities metropolitan area.

Individuals in the IDMI group began therapy 1 week after baseline assessment and were seen weekly for 6 sessions. One week after the final session, they returned for evaluation. Individuals assigned to GA returned after 8 weeks for follow-up. People in this group were given six sessions of IDMI beginning 1 week following the 8-week post-GA assessment. The primary outcome measure was the Yale-Brown Obsessive Compulsive Scale Modified for Pathological Gambling (PG-YBOCS), ${ }^{4}$ a 10 -item scale comprising an urge/thought subscale and a behaviour subscale. Initial treatment response between those assigned to the IDMI and GA groups were compared by analysis of covariance (ANCOVA). The baseline measure of the end-point was entered as a covariate in the model. Within-participant differences for the GA group between post- 8 weeks of GA and post-IDMI treatment were tested using the baseline measure as a covariate in an intent-to-treat ANCOVA repeated-measures model. Using previously reported effect sizes from other psychosocial studies of pathological gambling $^{2}$ and a two-tailed alpha level of 0.05 , a total sample of 68 was required to generate a power of 0.80 .

\section{Results}

Sixty-eight participants (43 (63.2\%) females, mean age 48.7 years $($ s.d. $=12.8))$ were randomised to IDMI $(n=33)$ or GA $(n=35)$ (online Fig. DS1). No statistically significant imbalances existed regarding any baseline variable. Individuals spent a mean of 12.5 hours (s.d. $=4.2$ ) each week gambling and had lost $49.7 \%$ of their gross income to gambling during the past year. Twenty-five $(75.8 \%)$ in the IDMI group and $30(85.7 \%)$ in the GA group completed the 8 -week treatment.

During the acute 8 -week treatment period, significantly better results on the PG-YBOCS were observed for the IDMI group (Table 1). Of the 33 participants in the IDMI group, 21 (63.6\%) were abstinent from all gambling for at least 1 month by the end of the 8-week period, whereas only 6 of the $35(17.1 \%)$ in the GA group were abstinent (Fisher's exact $<0.001$ ). Twenty-six individuals assigned to GA attended at least one meeting (mean weekly attendance 1.1 meetings $($ s.d. $=2.4)$ ).

Participants in the IDMI group demonstrated a significantly greater response to the other measures of gambling severity (G-SAS, CGI-S), depression and anxiety symptoms and psychosocial functioning (i.e. SDS score) (Table 1). There were no significant gender differences in treatment response.

Those initially assigned to GA showed a statistically greater reduction in gambling symptoms on the PG-YBOCS after receiving IDMI following 8 weeks of GA $(F(1,26)=27.192$, $P<0.001)$. Significant symptom improvement following IDMI 


\begin{tabular}{|c|c|c|c|c|c|c|c|}
\hline \multirow[b]{2}{*}{ Variable } & \multicolumn{2}{|c|}{ Baseline } & \multicolumn{2}{|c|}{ 8-week end-point (LOCF)a } & \multirow[b]{2}{*}{$F(1,65)^{\mathrm{b}}$} & \multirow[b]{2}{*}{$P$} & \multirow[b]{2}{*}{$\begin{array}{l}\text { Cohen's } \\
\text { effect size }\end{array}$} \\
\hline & $\begin{array}{c}\text { IDMI group } \\
(n=33), \text { mean (s.d.) }\end{array}$ & $\begin{array}{c}\text { GA group } \\
(n=35), \text { mean (s.d.) }\end{array}$ & $\begin{array}{c}\text { IDMI group } \\
(n=33), \text { mean (s.d.) }\end{array}$ & $\begin{array}{c}\text { GA group } \\
(n=35), \text { mean (s.d.) }\end{array}$ & & & \\
\hline \multicolumn{8}{|l|}{ PG-YBOCS } \\
\hline Total score & $19.1(5.45)$ & $19.7 \quad(5.81)$ & $9.00(7.78)$ & $18.1(7.70)$ & 48.31 & $<0.001$ & 1.55 \\
\hline Urge/thought subscale & $9.18(3.28)$ & $9.40(3.84)$ & $5.48(3.91)$ & $9.40(3.85)$ & 23.40 & $<0.001$ & 1.06 \\
\hline Behaviour subscale & $9.94(4.32)$ & $10.3(4.34)$ & $3.52(5.11)$ & $8.63(5.47)$ & 17.78 & $<0.001$ & 1.13 \\
\hline G-SAS ${ }^{5}$ total score & $29.3(7.70)$ & $30.2(7.99)$ & $19.9(11.2)$ & $29.7(10.6)$ & 20.25 & $<0.001$ & 1.14 \\
\hline $\mathrm{CGI}{ }^{6}$ - Severity & $4.70(0.68)$ & $4.71(0.83)$ & $2.73(1.44)$ & $4.46(1.17)$ & 31.48 & $<0.001$ & 2.26 \\
\hline $\mathrm{HRSD}^{7}$ & $6.36(5.11)$ & $7.60(4.71)$ & $4.64(4.83)$ & $8.00(4.99)$ & 7.76 & 0.007 & 0.52 \\
\hline $\mathrm{HAS}^{8}$ & $4.88(3.92)$ & $6.20(3.75)$ & $3.73(3.80)$ & $6.37(3.51)$ & 7.12 & 0.010 & 0.47 \\
\hline Sheehan Disability Scale ${ }^{9}$ & $11.94(7.03)$ & $14.69(7.92)$ & $5.39(6.14)$ & $12.49(8.41)$ & 13.50 & $<0.001$ & 0.74 \\
\hline Quality of Life Inventory ${ }^{10}$ & 36.6 (13.5) & $31.4(16.1)$ & 40.9 (12.4) & $32.6(15.9)$ & 3.66 & 0.060 & 0.31 \\
\hline \multicolumn{8}{|c|}{$\begin{array}{l}\text { LOCF, last observation carried forward (when visit data missing); PG-YBOCS, Yale-Brown Obsessive Compulsive Scale Modified for Pathological Gambling; G-SAS, Gambling Symptom } \\
\text { Assessment Scale; CGI-S, Clinical Global Impression - Severity Scale; HRSD, Hamilton Rating Scale for Depression; HAS, Hamilton Anxiety Scale. } \\
\text { a. Imaginal desensitisation plus motivational interviewing and Gamblers Anonymous adjusted for baseline level. } \\
\text { b. ANCOVA F-test for group assignment. } \\
\text { c. Cohen's effect size based on differences in covariate adjusted mean differences. }\end{array}$} \\
\hline
\end{tabular}

was also observed on the PG-YBOCS urge subscale $(F(1,26)=22.188, \quad P<0.001), \quad$ PG-YBOCS behaviour subscale $(F(1,26)=22.277, P<0.001)$, G-SAS $(\mathrm{F}(1,26)=14.359, P=0.001)$, CGI-S $\quad(F(1,26)=17.199, \quad P<0.001), \quad$ SDS $\quad(F(1,26)=15.529$, $P=0.001)$ and $\operatorname{QoLI}(F(1,26)=10.222, P=0.004)$.

\section{Discussion}

We found IDMI to be superior to GA referral in acute treatment of pathological gambling across a spectrum of illness-specific and global outcome measures. The results demonstrate that this treatment reduces pathological gambling symptoms and is effective in improving measures of psychosocial functioning and quality of life.

Manualised IDMI was effective in reducing gambling urges as well as behaviour. Unlike conventional treatment, where the individual may not experience urges to gamble during a therapy session, this treatment elicits gambling urges throughout the day and provides, via audiotapes, the immediate cognitive restructuring to control the urges. One theory for its effectiveness is that impairments in prefrontally mediated cognitive functions appear to underlie behavioural dysregulation, namely decision-making and inhibitory control. ${ }^{11}$ These impairments may increase the risk for making impulsive decisions that focus on short-terms gains. Treatment with IDMI allows individuals to experience the urge and immediately increases inhibitory control by focusing on decisions that consider both short- and long-term behavioural consequences.

This study also shows that the most commonly used treatment intervention, GA, was beneficial in only a minority of cases in the short term. Although some long-term benefits from GA have been documented, ${ }^{1,2}$ the limited short-term benefits seen in this study may explain the high rates of treatment discontinuation. ${ }^{12}$ However, our exclusion of individuals who had previously attended GA may have resulted in poor GA attendance during the study and worse overall outcomes.

This study has several limitations. First, it is unclear how many IDMI sessions are optimal. It is possible that a longer course of therapy could result in continued and even greater reductions in gambling symptoms. Second, individuals assigned to GA attended meetings very infrequently, so we cannot determine whether GA would be more efficacious if attended more regularly. Finally, although the GA group demonstrated significant benefit from later assignment to IDMI, we cannot rule out a possible impact of earlier GA attendance on later IDMI treatment.

This investigation suggests that IDMI may be effective in the treatment of pathological gambling. As effective treatments for pathological gambling emerge, it becomes increasingly important that physicians and mental healthcare providers screen for pathological gambling in order to provide timely treatment.

Jon E. Grant, JD, MD, MPH, Christopher B. Donahue, PhD, Brian L. Odlaug, BA Suck Won Kim, MD, Michael J. Miller, PhD, Department of Psychiatry, University of Minnesota School of Medicine, Minneapolis; Nancy M. Petry, Department of Psychiatry, University of Connecticut Health Center, Farmington, Connecticut, USA

Correspondence: Jon E. Grant, Department of Psychiatry, University of Minnesota School of Medicine, 2450 Riverside Avenue, Minneapolis, MN 55454 USA. Email: grant045@umn.edu

First received 28 Nov 2008, accepted 5 Mar 2009

\section{Funding}

Supported by an NIMH Career Development Award (K23 MH069754-01A1) (J.E.G.)

\section{References}

1 Petry NM. Pathological Gambling: Etiology, Comorbidity, and Treatment. American Psychological Association, 2005.

2 Hodgins DC, Petry NM. Cognitive and behavioral treatments. In Pathological Gambling: A Clinical Guide to Treatment (eds JE Grant, MN Potenza): 169-87. APPI, 2004.

3 Grant JE, Steinberg MA, Kim SW, Rounsaville BJ, Potenza MN. Preliminary validity and reliability testing of a structured clinical interview for pathological gambling (SCI-PG). Psychiatry Res 2004; 128: 79-88.

4 Pallanti S, DeCaria CM, Grant JE, Urpe M, Hollander E. Reliability and validity of the Pathological Gambling Modification of the Yale-Brown ObsessiveCompulsive Scale (PG-YBOCS). J Gambling Stud 2005; 21: 431-43.

5 Kim SW, Grant JE, Potenza MN, Blanco C, Hollander E. The Gambling Symptom Assessment Scale (G-SAS): a reliability and validity study. Psychiatry Res, 2009; 166, 76-84.

6 Guy W. ECDEU Assessment Manual for Psychopharmacology. US Dept Health, Education and Welfare publication (ADM) 76-338. National Institute of Mental Health, 1976

7 Hamilton M. A rating scale for depression. J Neurol Neurosurg Psychiatry 1960; 23: 56-62.

8 Hamilton M. The assessment of anxiety states by rating. Br J Med Psychol 1959; 32: $50-5$

9 Sheehan DV. The Anxiety Disease. Scribner's, 1983.

10 Frisch MB, Cornell J, Villaneuva M. Clinical validation of the Quality of Life Inventory: a measure of life satisfaction for use in treatment planning and outcome assessment. Psychol Assess 1993; 4: 92-101.

11 Yucel M, Lubman DI. Neurocognitive and neuroimaging evidence of behavioural dysregulation in human drug addiction: implications for diagnosis, treatment and prevention. Drug Alcohol Rev 2007; 26: 33-9.

12 Stewart RM, Brown RI. An outcome study of Gamblers Anonymous. Br J Psychiatry 1988; 152: 284-8. 